\title{
Motion Style Acupuncture Treatment for Acute Lumbar Sprain: a Protocol for Systematic Review and Meta-analysis
}

\section{Ju Wang}

Chengdu University of TCM: Chengdu University of Traditional Chinese Medicine https://orcid.org/0000-0002-9684-4288

Di Zhang

Chengdu University of Traditional Chinese Medicine Affiliated Hospital

Tianyu Zhao

CDUTCM: Chengdu University of Traditional Chinese Medicine

Jiang Ma

CDUTCM: Chengdu University of Traditional Chinese Medicine

Xiaoxiao Liu

Chengdu University of TCM: Chengdu University of Traditional Chinese Medicine

\section{Yinli Shen}

Chengdu University of Traditional Chinese Medicine Affiliated Hospital

\section{Danqing Luo}

Chengdu University of Traditional Chinese Medicine Affiliated Hospital

\section{Hong Guo}

Chengdu University of Traditional Chinese Medicine Affiliated Hospital

\section{Song Jin ( $\sim$ j1049147000@163.com )}

Chengdu University of Traditional Chinese Medicine Affiliated Hospital https://orcid.org/0000-00034725-780X

\section{Protocol}

Keywords: Acute lumbar sprain, motion style acupuncture treatment, acupuncture, motion therapy, randomized controlled trial

Posted Date: January 15th, 2021

DOI: https://doi.org/10.21203/rs.3.rs-146106/v1

License: (c) (i) This work is licensed under a Creative Commons Attribution 4.0 International License. Read Full License 


\section{Abstract}

Background: Acute lumbar sprain is a common cause of acute low back pain. Motion style acupuncture treatment (MSAT; a combination of acupuncture and motion therapy) has been used for patients with acute lumbar sprain, but evidence of its efficacy is inconsistent. The aim of this systematic review with meta-analysis is to determine the effectiveness and security of motion style acupuncture treatment (MSAT) for acute lumbar sprain and to determine whether long-term effectiveness exists.

Methods: Eight databases will be searched, including PubMed, SoniMed, Cochrane Library, EMBAS, Springer, China National Knowledge Infrastructure, Wanfang date, and Chinese Biomedical Literature Database from inception to February 1, 2021, will be restricted to English and Chinese. Methodologic quality was independently screened and graded by two blind reviewers using the Cochrane Collaborative bias risk tool. Meta-analysis was performed in at least three studies, and sub-group and sensitivity analyses were conducted when $I^{2}>50 \%$. When the quantitative evaluation is not available, we will provide a qualitative description of the results of the individual study.

Discussion: The conclusion of this protocol will provide evidence to judge the potential benefits of motion style acupuncture and conduct clinical decision-making for acute lumbar sprain treatment.

Systematic review registration: A protocol had been registered in PROSPERO CRD42020192876.

\section{Background}

Acute lumbar sprain is a common musculoskeletal disease caused by acute injury of the lumbar muscles, ligaments, fasciae, or intervertebral facet joints. [1] Studies have shown that the morbidity of this disease accounts for about $12 \%$ of lumbago disease with an increasing incidence, which affects $60 \%-80 \%$ of adults. [2-4] The disease can occur at any age, but clinical manifestations are mainly in the young and middle-aged groups.[5] The main clinical manifestations are acute low back pain, difficulty in lumbar activity, lumbar muscle stiffness and myo-spasm, and back pain is affected by position transfer, cough, sneezing, and breathing.[6] The main pathogenesis of acute lumbar sprain is associated with stress congestion and exudation that increases permeability of the capillary wall appears in local soft tissue, the accumulation of inflammatory pain substances causes microcirculation disorders, stimulating nerve endings lead to pain, swelling, and limited activity.[7] It could turn into a chronic disease, if not treated and managed in a timely manner.[8] Worse still, it might affect people's quality of life, social participation, and increase the cost of medical care and imposes a socioeconomic burden.[9-11] Therefore, acute lumbar sprain has gained increasing attention.

The main therapeutic objective of acute low back pain is to control pain and maintain function.[12] According to the 2020 US guidelines, nonsteroidal anti-inflammatory drugs (NSAIDs), skeletal muscle relaxants, benzodiazepines and physical therapy are commonly used to treat acute lumbar sprain.[13] However, drug safety and patient satisfaction are controversial for the side effects of nonsteroidal antiinflammatory drugs (NSAIDs) in the gastrointestinal and cardiovascular systems.[14] Some guidelines 
recommend pharmacological therapy only if non-pharmacological treatment does not succeed in patients with acute and subacute low back pain.[15-16] Thus, it is necessary to explore effective, safe, and economical non-pharmacological therapies.

Acupuncture is an important non-pharmacological treatment that has been used to treat diseases for more than 3000 years and was spread to Europe and American from the sixteenth to the nineteenth century.[17] Motion style acupuncture treatment (MSAT) is a relatively novel acupuncture method that is characterized by the combination of acupuncture with motion therapy. Patients are required to perform local exercises with acupuncture needles in the distal limbs.[18] Motion style acupuncture treatment (MSAT) as one of the complementary and alternative medical therapies for acute lumbar sprain, several studies have shown that it could improve acute lumbar sprain symptoms by relieving pain and improving lumbar activity.[19-20] According to the theory of traditional Chinese medicine, which relieves pain by removing the obstruction to the flow of Qi.[21-23] Studies have shown that the De Qi sensation could induce the release of a variety of neurotransmitters during acupuncture.[24-25] Acupuncture activates acupoints with special anatomical structures and transmit signals that activate neurotransmitters, and regulators such as endorphins, 5-hydroxytryptamines, and dopamines, which have an analgesic effect during acupuncture.[26] Local lumbar exercise can improve lumbar activity, which may be able to increase the feel of De Qi, and improve the therapeutic effect.[27] However, due to the variety of acupuncture approaches, the relative effectiveness was inconsistent.

Two previous systematic reviews have discussed the efficacy of acupuncture for acute lumbar sprain in 2016 and 2020.[28-29] However, they focused on the efficacy of simple acupuncture therapy for acute lumbar sprain instead of motion style acupuncture. Another review of 2015 evaluated acupuncture combined therapy (acupuncture with exercise) with insufficient sample size, deficiency of lumbar activity, and long-term efficacy in low-quality literature.[30] Thus, in this review, we will evaluate the efficacy and security of motion style acupuncture treatment for a definite conclusion for acute lumbar sprain and determine whether long-term effectiveness exists, which will be based on latest RCTs, and provide new evidence for clinical selection.

\section{Methods /design}

\section{The aim and objective}

This systematic review protocol aims to determine the efficacy and securityof Motion style acupuncture treatment for acute lumbar sprain.

\section{Study design}

The systematic review has been registered on PROSPERO with a registration number of CRD42020192876. This study will be conducted in accordance with the Preferred Reporting Items for Systematic Reviews and Meta-Analyses protocols (PRISMA-P) checklist (see Additional file 1 for 
completed checklist) [31] and will be conducted in accordance with the Cochrane Handbook for Systematic Reviews of Interventions.[32]

\section{Information sources and search trategy}

The related databases including PubMed, SoniMed, Cochrane Library, EMBAS, Springer, China National Knowledge Infrastructure(CNKI), Wanfang data, and Chinese Biomedical Literature Database(CBM) will be searched. All RCTs published in electronic databases from inception to February 1st, 2021 with language restricted in Chinese and English will be included. Medical Subject Headings (MeSH) and variants for"acute lumbar sprain", "acupuncture", and "motion therapy"will be used and combined in the searches. The primary selection process is shown in the PubMed search strategy (see table 1).

\section{Eligibility criteria}

\section{Types of studies}

Randomized controlled trials (RCTs) of motion style acupuncture treatment (MAST) for acute lumbar sprain. A randomized study was conducted if the trial stated the"randomization"phrase, and blinding was not restricted. The case reports, animal mechanism studies, self-pre and post-control, expert experience, duplicated publications, or non-RCTs will be excluded.

\section{Types of participants}

\section{Inclusion criteria}

According to the guidelines for diagnosis and treatment of low back pain (2019)[33], participants(aged $\geq$ 18 years) of acute lumbar sprain with 1 . history of acute lumbar sprain; 2 . often occurs in the low back segment; 3 . acute low back pain and limited activity; 4 .obvious pain points, muscle spasm, and stiffness will be included.

\section{Exclusion criteria}

Participants with nerve sexual pain, vertebral infection, neoplastic low back pain, low back pain from internal viscera, spinal fracture dislocation, acute medical conditions, and pregnant women will be excluded on account of actual clinical conditions.

\section{Types of interventions}

Motion style acupuncture treatment (MSAT) in this study included all types of acupuncture combined with motion threads, such as electroacupuncture, dry-needle, auriculotherapy, superfcial needling, acupressure, abdominal acupuncture, bleeding therapy, warm acupuncture, blade needle, eye acupuncture, needle knife, and so on. Studies comparing the effects of different acupuncture treatments will be excluded. 


\section{Types of control groups}

The intervention groups included pure acupuncture therapy, exercise therapy, pharmacotherapy, shamacupuncture, placebo, or no additional intervention to usual care. Studies that compared different acupoints will be excluded.

\section{Types of outcome measures}

Studies will include one or more of the following outcomes.

\section{The primary outcomes:}

1. Effective rate.

2. The change in pain intensity was identified using a visual analogue scale (VAS) or numeric rating scale (NRS).

3. Functional outcome measures were identified using the range of motion (ROM).

\section{The Secondary outcomes:}

1. Quality of life measured by validated scales includes SF-36 or other validated outcome measures.

2. Dysfunction questionnaire score using oswestry disability index (ODI).

3. Treatment frequency and courses.

4. Side effects, including pneumothorax, bleeding, serious discomfort, subcutaneous nodules, and infection, will be recorded.

5. The number of participants dropped out, and the number of participants reporting adverse events was also recorded.

\section{Data collection and analysis}

Two independent reviewers( JW and DZ) independently will scan the titles and abstracts according to the inclusion criteria and screen articles by their full-text version. They will extract the data into a selfdesigned data extraction form if it satisfies the inclusion and exclusion criteria; finally, they will crosscheck the selection results. Any disagreements will be arbitrated by a third reviewer (SJ). The selection procedure is shown in a PRISMA flow diagram (see Additional file 2).

\section{Data extraction and management}

The data extraction form will include basic information (article title, first author, publication year, country, mean age of the population, number of males and females), study design (RCT design, blind method, randomization, sample size), interventions (including the methods, frequency and duration of interventions), outcome measures, adverse effects, duration of follow-up, conclusions, and source of financial support according to the Standards for Reporting Interventions in Controlled Trials of 
Acupuncture (STRICTA) checklist.[34] The completed data extraction forms were cross-checked by the two reviewers. Any diversity will be resolved by a third reviewer (SJ).

\section{Assessment of risk of bias and quality}

Two reviewers (JW and JM) will assess the risk of bias of included trials by the Cochrane Collaboration's risk of bias assessment method. We assessed the risk of bias (low, high and unclear risk) in six areas including performance bias (blinding of participants and personnel), selection bias (random sequence generation, allocation concealment), detection bias (blinding of outcome assessment), attrition bias (incomplete outcome data), reporting bias (selective reporting), and other biases. For duplicate publications, we only select the original. The final decisions will be made by the third reviewer (JS) if inconsistent results appear.

\section{Measures of treatment effect}

The mean differences (MDs) with 95\% confidence intervals (95\% Cls) were used for continuous data. Other forms of data will be changed to MD values. The risk ratio with $95 \% \mathrm{Cls}$ will be used to analyze dichotomous outcomes. Ordinal outcomes (e.g., 'almost cured, remarkably effective, effective, or 'not effective') in two or more categories will be transformed into dichotomous outcomes such as responders and non-responders. A random-effects mode will be used if significant heterogeneity is detected.

\section{Management of missing data}

For incomplete data or missing data, we will contact the corresponding author to request additional information. If we not get the incomplete data or missing data, it will be excluded from analysis.

\section{Assessment of heterogeneity}

Review Manager software (V.5.3) will be used to assess the curative effect and publication bias. The relative strength of the curative effect will use the Forest plot to illustrate. Meanwhile, if trials are more than 10 , the funnel plot will be used to assess the publication bias visually. In addition, subgroup analyses or meta-regression will be used to explore the source of heterogeneity.

\section{Data analysis}

We will use the Review Manager (V.5.3) for data analysis if there are two or more studies of sufficient homogeneity across the outcome measures to ensure pooling. Heterogeneity was analyzed using the $\mathrm{I}^{2}$ statistic. The RR and MD will be calculated by a fixed-effects model, if $\mathrm{I}^{2}<50 \%$. A random-effects model will be used to synthesize the data, if $\mathrm{I}^{2} \geq 50 \%$. We will use subgroup analysis or sensitivity analysis to explore the causes of heterogeneity such as clinical or methodological reasons. When the quantitative evaluation is not available, we will provide a qualitative description of the results of the individual study.

\section{Narrative analysis}


Narrative synthesis will be used if meta-analysis is not appropriate (e.g., incidence of adverse events of acupuncture).

\section{Subgroup analysis}

We will conduct a subgroup analysis according the control intervention and different results.

\section{Sensitivity analysis}

Sensitivity analysis will be conducted according to several nodes such as methodological weaknesses, sample size, missing data, and statistical models.

\section{Grading the quality of evidence}

The quality of evidence for outcomes will be independently assessed by two reviewers using the Grading of Recommendations Assessment, Development and Evaluation guidelines (GRADE).[35] The strength of the body of evidence is divided into four levels: high, medium, low, and very low. [36]

\section{Discussion}

Motion style acupuncture treatment (MSAT) is a relatively novel acupuncture method. Currently, it is used to treat acute lumbar sprain in the oriental world, which has increased as it enhances the effectiveness of traditional acupuncture treatment.[37] The immediate effect of acupuncture may lay the foundation for motion, which enhances the curative effect of single acupuncture and long-term effects.[38] However, there is limited high-quality evidence regarding the efficacy and safety of motion style acupuncture treatment (MSAT). Thus, in order to better understand the potential benefits of acupuncture combined with motion therapy and guide clinical decision-making, we will design this study to determine the effectiveness and safety of motion style acupuncture treatment (MSAT) for acute lumbar sprain according to the latest RCTs.

In this study, a large number of original studies will be collected to evaluate the efficacy and safety of motion style acupuncture treatment (MSAT) for lumbar sprain. The results may be affected by potential limitations. Language limitation of English and Chinese may result in a risk of bias in some kind. The variety of acupuncture protocols used in the RCT review may result in a risk of heterogeneity and finally influence the conclusion of this review, and new, unpublished data may not be available at the time of submission of this review.

Thus, in order to better understand the potential benefits of motion style acupuncture and conduct clinical decision-making for acute lumbar sprain treatment, various outcomes are expected to be explored in each study and measured over time.

\section{List Of Abbreviations}


MSAT, motion style acupuncture treatment; Cl, confidence interval; MD, mean difference; PRISMA-P, preferred reporting items for systematic reviews and meta-analysis protocol; RCT, randomized controlled trial; RR, risk ratio; VAS, visual analog scale; NRS, numeric rating scale; ROM, range of motion; ODI, Oswestry disability index.

\section{Declarations}

\section{Ethics approval and consent to participate:}

Ethical approval is not required for literature-based studies. The results will be disseminated through peerreviewed publications.

\section{Patient consent for publication:}

Not required.

\section{Availability of data and materials:}

Not applicable.

\section{Consent for publication:}

Not applicable.

\section{Competing interests:}

The authors have no conflicts of interest to disclose.

\section{Provenance and peer review:}

Not commissioned; externally peer reviewed.

\section{Funding:}

This study was supported by the Department of Science and Technology of Sichuan Province (Grant NO.2020YFS0381).

\section{Author Contributions:}

JW and DZ contributed equally to this study. JW conceived this study and developed the first frame of this manuscript. JW and JM drafted the manuscript. JW, TYZ, and XXL contributed to the development of the search strategy, YLS performed data collection, DQL and HG read the full texts of studies and extracted data. SJ, DZ revised the manuscript, and SJ provided funding support. All authors read and approved the final manuscript.

\section{Acknowledgements:}


Not applicable.

\section{References}

1. Chou R, Qaseem A, Snow V, Casey D, Cross JT, Jr., Shekelle P, et al. Diagnosis and treatment of low back pain: a joint clinical practice guideline from the American College of Physicians and the American Pain Society. Annals of internal medicine. 2007;147(7):478-91. doi: 10.7326/0003-4819147-7-200710020-00006.

2. Rubin DI. Epidemiology and risk factors for spine pain. Neurologic clinics. 2007;25(2):353-71. doi: 10.1016/j.ncl.2007.01.004.

3. Bernardes F, Mendes-Castro A, Ramos J, Costa O. Musculoskeletal Injuries in Competitive Rowers. Acta medica portuguesa. 2015;28(4):427-34.

4. Panagodage Perera NK, Kountouris A, Kemp JL, Joseph C, Finch CF. The incidence, prevalence, nature, severity and mechanisms of injury in elite female cricketers: A prospective cohort study. Journal of science and medicine in sport. 2019;22(9):1014-20. doi: 10.1016/j.jsams.2019.05.013.

5. Fan Y, Wu Y. Effect of electroacupuncture on muscle state and infrared thermogram changes in patients with acute lumbar muscle sprain. Journal of traditional Chinese medicine. 2015;35(5):499506. doi: 10.1016/s0254-6272(15)30131-x.

6. Liang S, Zhang G, Li J, Zhong L, Zhang C. Wrist ankle acupuncture in the treatment of acute lumbar sprain: A protocol for systematic review and meta-analysis. Medicine. 2020;99(49):e23420. doi: 10.1097/md.0000000000023420.

7. Liu LL, Lu J, Ma HF. Clinical Trials for Treatment of Acute Lumbar Sprain by Acupuncture Stimulation of "Yaotong" and Local Ashi-points in Combination with Patients' Lumbar Movement. Acupuncture research. 2017;42(1):72-5.

8. Rolli Salathé C, Melloh M, Mannion AF, Tamcan Ö, Müller U, Boos N, et al. Resources for preventing sickness absence due to low back pain. Occupational medicine (Oxford, England). 2012;62(4):27380. doi: $10.1093 /$ occmed/kqs024.

9. Vasseljen O, Woodhouse A, Bjørngaard JH, Leivseth L. Natural course of acute neck and low back pain in the general population: the HUNT study. Pain. 2013;154(8):1237-44. doi: 10.1016/j.pain.2013.03.032.

10. Verbunt JA, Sieben JM, Seelen HA, Vlaeyen JW, Bousema EJ, van der Heijden GJ, et al. Decline in physical activity, disability and pain-related fear in sub-acute low back pain. European journal of pain (London, England). 2005;9(4):417-25. doi: 10.1016/j.ejpain.2004.09.011.

11. Dagenais S, Caro J, Haldeman S. A systematic review of low back pain cost of illness studies in the United States and internationally. The spine journal : official journal of the North American Spine Society. 2008;8(1):8-20. doi: 10.1016/j.spinee.2007.10.005.

12. Miller SM. Low back pain: pharmacologic management. Primary care. 2012;39(3):499-510. doi: 10.1016/j.pop.2012.06.005. 
13. Qaseem A, McLean RM, O'Gurek D, Batur P, Lin K, Kansagara DL. Nonpharmacologic and Pharmacologic Management of Acute Pain From Non-Low Back, Musculoskeletal Injuries in Adults: A Clinical Guideline From the American College of Physicians and American Academy of Family Physicians. Annals of internal medicine. 2020;173(9):739-48. doi: 10.7326/m19-3602.

14. Busse JW, Craigie S, Sadeghirad B, Couban R, Hong P, Oparin Y, et al. Management of acute musculoskeletal pain (excluding low back pain): protocol for a systematic review and network metaanalysis of randomised trials. BMJ open. 2019;9(4):e024441. doi: 10.1136/bmjopen-2018-024441.

15. Qaseem A, Wilt TJ, McLean RM, Forciea MA. Noninvasive Treatments for Acute, Subacute, and Chronic Low Back Pain: A Clinical Practice Guideline From the American College of Physicians. Annals of internal medicine. 2017;166(7):514-30. doi: 10.7326/m16-2367.

16. Oliveira CB, Maher CG, Pinto RZ, Traeger AC, Lin CC, Chenot JF, et al. Clinical practice guidelines for the management of non-specific low back pain in primary care: an updated overview. Eur Spine J. 2018;27(11):2791-803. doi: 10.1007/s00586-018-5673-2.

17. Zhuang Y, Xing JJ, Li J, Zeng BY, Liang FR. History of acupuncture research. International review of neurobiology. 2013;111:1-23. doi: 10.1016/b978-0-12-411545-3.00001-8.

18. Lee SH, Lee J, Lee YJ, Kim MR, Cho JH, Kim KW, et al. Effectiveness and cost-effectiveness of acupuncture with Doin therapy for chronic neck pain: a study protocol for a multicentre, randomised controlled clinical trial. BMJ open. 2019;9(5):e026632. doi: 10.1136/bmjopen-2018-026632.

19. Magrinelli F, Roncari L, Tamburin S. 'Effects of motion style acupuncture treatment in acute low back pain patients with severe disability' by Shin et al. Pain. 2014;155(1):201-2. doi: 10.1016/j.pain.2013.08.020.

20. Shin JS, Ha IH, Lee J, Choi Y, Kim MR, Park BY, et al. Effects of motion style acupuncture treatment in acute low back pain patients with severe disability: a multicenter, randomized, controlled, comparative effectiveness trial. Pain. 2013;154(7):1030-7. doi: 10.1016/j.pain.2013.03.013.

21. Salih N, Bäumler PI, Simang M, Irnich D. Deqi sensations without cutaneous sensory input: results of an RCT. BMC complementary and alternative medicine. 2010;10:81. doi: 10.1186/1472-6882-10-81.

22. Yang XY, Shi GX, Li QQ, Zhang ZH, Xu Q, Liu CZ. Characterization of deqi sensation and acupuncture effect. Evid Based Complement Alternat Med. 2013;2013:319734. doi: 10.1155/2013/319734.

23. Deadman P, Al-Khafaji M, Baker K. A manual of acupuncture. 3rd ed. J Chin Med. Beijing: 2008. P. 574-580.

24. Chen T, Zhang WW, Chu YX, Wang YQ. Acupuncture for Pain Management: Molecular Mechanisms of Action. The American journal of Chinese medicine. 2020;48(4):793-811. doi: $10.1142 / \mathrm{s} 0192415 \times 20500408$.

25. Li M, Yuan H, Wang P, Xin S, Hao J, Liu M, et al. Influences of De Qi induced by acupuncture on immediate and accumulated analgesic effects in patients with knee osteoarthritis: study protocol for a randomized controlled trial. Trials. 2017;18(1):251. doi: 10.1186/s13063-017-1975-7.

26. Wang SM, Kain ZN, White P. Acupuncture analgesia: I. The scientific basis. Anesthesia and analgesia. 2008;106(2):602-10. 
27. Lin R, Zhu N, Liu J, Li X, Wang Y, Zhang J, et al. Acupuncture-movement therapy for acute lumbar sprain: a randomized controlled clinical trial. Journal of traditional Chinese medicine. 2016;36(1):1925. doi: 10.1016/s0254-6272(16)30003-6.

28. Yu X. Clinical efficacy of acupuncture on acute lumbar sprain: Meta-analysis. Chengdu:University of Chinese Medicine; 2020.

29. Li J. Acupuncture treatment of acute lumbar sprain in recent 10 years : Systematic review and Metaanalysis. Dissertation. Beijing: Beijing University of Chinese Medicine; 2016.

30. HeX. Acupuncture therapy combined with exercise treatment acute lumbar sprain: Systematic review. Chengdu:Chengdu University of TCM; 2015.

31. Shamseer L, Moher D, Clarke M, Ghersi D, Liberati A, Petticrew M, et al. Preferred reporting items for systematic review and meta-analysis protocols (PRISMA-P) 2015: elaboration and explanation. Bmj. 2015;350:g7647. doi: 10.1136/bmj.g7647.

32. Shamseer L, Moher D, Clarke M, Ghersi D, Liberati A, Petticrew M, et al. Preferred reporting items for systematic review and meta-analysis protocols (PRISMA-P) 2015: elaboration and explanation. Bmj. 2015;350:g7647. doi: 10.1136/bmj.g7647.

33. Higgins JPT, Green S. Cochrane Handbook for Systematic Reviews of Interventions Version 5.1.0. British: The Cochrane Collaboration; 2011.

34. Pangarkar SS, Kang DG, Sandbrink F, Bevevino A, Tillisch K, Konitzer L, et al. VA/DoD Clinical Practice Guideline: Diagnosis and Treatment of Low Back Pain. Journal of general internal medicine. 2019;34(11):2620-9. doi: 10.1007/s11606-019-05086-4.

35. MacPherson H, Altman DG, Hammerschlag R, Youping L, Taixiang W, White A, et al. Revised STandards for Reporting Interventions in Clinical Trials of Acupuncture (STRICTA): extending the CONSORT statement. PLoS medicine. 2010;7(6):e1000261. doi: 10.1371/journal.pmed.1000261.

36. Guyatt GH, Oxman AD, Vist GE, Kunz R, Falck-Ytter Y, Alonso-Coello P, et al. GRADE: an emerging consensus on rating quality of evidence and strength of recommendations. Bmj. 2008;336(7650):924-6. doi: 10.1136/bmj.39489.470347.

37. Guyatt GH, Oxman AD, Schünemann HJ, Tugwell P, Knottnerus A. GRADE guidelines: a new series of articles in the Journal of Clinical Epidemiology. Journal of clinical epidemiology. 2011;64(4):380-2. doi: 10.1016/j.jclinepi.2010.09.011.

38. Shi GX, Liu BZ, Wang J, Fu QN, Sun SF, Liang RL, et al. Motion style acupuncture therapy for shoulder pain: a randomized controlled trial. Journal of pain research. 2018;11:2039-50. doi: 10.2147/jpr.s161951.

\section{Table}

Table 1

Search strategies applied to this review. 
Number

$\# 1$

$\# 2$

\#3

$\# 4$

$\# 5$

\#6

$\# 7$

$\# 8$

$\# 9$

$\# 10$

\#11

\#12

\#13

\#14

$\# 15$

\#16

\#17

\#18

$\# 19$

\#20

\#21

\#22

\#23

\#24

\#25

\#26

\#27

\author{
Search items \\ Acupuncture \\ Acupoint \\ Meridian \\ Electroacupuncture \\ Dry-needle \\ Auriculotherapy \\ Superfical needling \\ Acupressure \\ Abdominal acupuncture \\ Bleeding \\ Warm acupuncture \\ Blade needle \\ Eye acupuncture \\ Needle Knife \\ OR/\#1-14 \\ Acute lumbar sprain \\ exercise threapy \\ motion threapy \\ Kinesithreapy \\ OR/\#17-19 \\ Randomized controlled trial \\ Controlled clinical trial \\ Randomly \\ Randomized \\ Trial \\ OR/\#21-25 \\ $\# 15$ and \#16and \#20and\#26
}

\section{Supplementary Files}

This is a list of supplementary files associated with this preprint. Click to download.

- Additionalfile1PRISMAP2015Checklist.docx

- Additionalfile2Flowdiagramofstudiesidentified..docx 